\title{
Les v[V]oix du Théâtre de l'Athénée dans les critiques dramatiques. Jalons pour une enquête
}

The $v[V]$ oices of the Athénée in Theatre Criticism: Investigative Milestones

\section{Tommaso Zaccheo}

\section{(2) OpenEdition}

\section{Journals}

Édition électronique

URL : https://journals.openedition.org/rsl/2085

DOI : $10.4000 /$ rsl.2085

ISSN : 2271-6246

Éditeur

Éditions Rue d'Ulm

Référence électronique

Tommaso Zaccheo, « Les v[V]oix du Théâtre de l'Athénée dans les critiques dramatiques. Jalons pour une enquête », Revue Sciences/Lettres [En ligne], 6 | 2019, mis en ligne le 10 décembre 2018, consulté le 30 juillet 2021. URL : http://journals.openedition.org/rsl/2085; DOI : https://doi.org/10.4000/rsl. 2085

Ce document a été généré automatiquement le 30 juillet 2021.

(c) Revue Sciences/Lettres 


\section{Les v[V]oix du Théâtre de l'Athénée dans les critiques dramatiques. Jalons pour une enquête}

The v[V]oices of the Athénée in Theatre Criticism: Investigative Milestones

Tommaso Zaccheo

1 En juin 2017, un petit chantier s'ouvrait à l'intérieur du vaste projet de recherche ECHO. L'objectif était de récolter un maximum d'informations concernant la dimension sonore des spectacles présentés au Théâtre de l'Athénée entre l'année 1945 et l'année 1973, période pour laquelle on ne dispose pas d'archives audio. Il s'agissait de se plonger, très concrètement, dans les revues de presse consacrées aux productions de ce théâtre parisien et, à l'aide de fiches techniques préalablement conçues, de savoir si et comment les commentateurs évoquaient les aspects sonores des représentations ${ }^{1}$. Dixneuf spectacles ont été choisis de façon à couvrir la période. Dans un premier temps, le travail a consisté à réaliser des fiches, une par spectacle, réunissant les informations générales sur la mise en scène et le relevé de tout ce qui concernait ses dimensions visuelles et sonores. Dans un deuxième temps ont été sélectionnés les passages où les critiques décrivaient le son, les voix, les bruits et la musique.

Cette enquête avait à la fois un caractère quantitatif - vu le nombre considérable de critiques à consulter - et qualitatif - vu l'attention toute particulière avec laquelle il fallait écouter ces témoignages. D'une part, le nombre de documents à analyser nous a obligé à nous concentrer sur trois grandes périodes de l'histoire de ce théâtre ${ }^{2}$. D'autre part, nous avons essayé de creuser ces sources, non seulement de façon à faire surgir le passé sonore de nos objets d'étude, mais aussi et surtout de façon à trouver des traces permettant de formuler des hypothèses concernant la qualité et l'évolution de l'écoute dans les critiques étudiées. En ce sens, nous avons essayé d'ériger en sources ces témoignages et de les "regarder historiquement", en suivant la définition que l'historien Antoine Prost donne de la «source historique " ${ }^{3}$. En bref, il s'agissait de considérer ces documents comme des traces d'un "passé auditif», tout en sachant, néanmoins, que celui-ci ne peut qu'être "présumé», comme l'indique Jonathan 
Sterne $e^{4}$. Durant la première phase du travail, cela a signifié extraire de ces critiques les passages relatifs au son, pour ensuite les comparer aux descriptions des autres éléments (éclairage, scénographie, etc.). Il ne suffisait pas de prendre en considération ce que les critiques disaient de la dimension sonore des spectacles, il fallait aussi noter ce qu'ils ne disaient pas, le langage qu'ils utilisaient et les possibles « traces du sonore » cachées derrière leurs commentaires. En un mot, nous étions à la recherche de l'auralité propre à la pratique de la critique dramatique à une période et dans un contexte précis, ce néologisme étant définissable comme

la combinaison de l'audible et de l'auditif, de ce qu'il y a à entendre et des protocoles (ce qui inclut les valeurs) à suivre pour entendre de façon optimale. On peut ainsi parler de l'auralité d'une pratique (le théâtre), d'une époque, d'une région ou d'une région à une époque, etc 5 .

3 La relecture synthétique des extraits sélectionnés montre que les informations récoltées et isolées peuvent révéler des aspects intéressants quant à l'écoute et à l'attention des « spectateurs professionnels » que sont les critiques envers le son de ces spectacles. Les lacunes de leurs descriptions et le silence envers l'acoustique constituant par ailleurs autant d'indices pour ouvrir d'autres pistes, pouvant conduire à une réflexion plus profonde sur la place du son dans le processus de réception des œuvres théâtrales.

4 Dans le cadre de cette étude, nous essayerons d'abord d'écouter un échantillon de ces documents textuels appréhendés comme des témoins de la dimension sonore des œuvres dont ils parlent. La distinction proposée par le philosophe Giorgio Agamben entre Voix et voix pourra être ici employée dans une perspective opératoire, selon une démarche dont Jeanne Bovet a déjà montré l'efficacitét. Il s'agira ensuite de vérifier ce que ces critiques ont écouté et ont su restituer dans un langage capable de relater leur expérience sonore. Enfin, nous essayerons de formuler des hypothèses concernant l'évolution du son et de sa perception à l'intérieur d'une "civilisation théâtrale ${ }^{7}$ " particulière, dans un arc chronologique de trente ans et à partir de ce corpus défini de mises en scène.

5 La Folle de Chaillot est le texte de Jean Giraudoux avec lequel Louis Jouvet ouvre la saison du Théâtre de l'Athénée après la Seconde Guerre mondiale ${ }^{8}$. Cet évènement est amplement préparé, documenté et commenté par la presse nationale, désireuse probablement d'un rapide « retour à la normalité » après les horreurs de l'Occupation. Henri Sauguet compose la musique (enregistrée), tout comme celle des productions les plus importantes de Jouvet après la guerre. Le 20 décembre 1945, il révèle lui-même au journal Paris-Presse avoir "tout simplement composé [...] une mazurka, chantée par toute la troupe, et une musique féerique pour la fin du deuxième acte ${ }^{9}$ ». Que reste-t-il de cette musique, conçue, semble-t-il, plutôt pour souligner deux moments clefs de la pièce que pour intervenir sur le sens de l'œuvre ? Presque rien, juste une ligne dans un article anonyme qui commente cette musique, sans pour autant la décrire. Dans $L a$ Bataille du 27 décembre 1946, nous pouvons en effet lire que « [...] M. Jouvet, lui, connaît l'art de fondre tous les éléments d'un spectacle, l'art de les conformer à l'esprit d'un texte et [que] le choix d'un musicien comme M. Sauguet pour une partition de dix minutes [confirme] ${ }^{10} »$. Si le travail de ce musicien est loué, il ne l'est qu'indirectement, puisque c'est le génie du chef d'orchestre Jouvet que l'on veut mettre en valeur, en soulignant son habileté à choisir ses collaborateurs et à "fondre» leur travail. La diction de tous les acteurs est en revanche abondamment commentée, celle de 
Marguerite Moreno (la Folle de Chaillot) et de Jouvet (le Chiffonnier) unanimement exaltée. René Bizet, par exemple, affirme dans Paris-Presse que Moreno,

[s]ans jamais tomber dans l'erreur du «ténor ", [...] sait, comme personne et parce qu'elle a un métier sans défaut, s'adresser au public tout en ayant l'air de causer avec ses partenaires dont elle ne se détache que lorsque les jeux de scène l'y contraignent. Résultat : tous les mots font balle, tout le texte de Giraudoux prend sa couleur, lance mille reflets et enchante l'oreille du public ${ }^{11}$.

La Voix de cette actrice semble être pour ce critique le premier des indices de sa maîtrise d'interprète, qui tout en étant capable de rester à l'écoute de ses partenaires sur scène, joue avec la salle, lui portant le texte de l'auteur. De la même manière, Bizet critique durement les jeunes acteurs "qui jouent pour eux ", " qui parlent vite, comme dans la conversation familière, s'adressent à leurs partenaires sans se préoccuper si leurs propos passent la rampe $»^{12}$. Ces observations semblent suggérer que dans le processus de réception/évaluation de ce spectacle, il est primordial que le verbe de Giraudoux soit transmis au public d'une façon compréhensible et que sa "couleur » propre soit mise en valeur. Cependant, leur conversation ne doit pas tomber dans le "familier », il faut que leur élocution projette les mots du texte tels des projectiles, afin que le verbe de Giraudoux puisse produire l'effet d'enchantement qui lui est propre. Cette pièce n'est perçue, au fond, que comme une " extraordinaire mélodie du verbe ${ }^{13}$ ", qui « réclame pour son service bien autre chose qu'une imagerie : un véritable concert des voix, minutieusement réglé, farouchement attentif à la nuance et au poids de chaque note ${ }^{14}$ ». C'est exactement le concert de la Voix de Moreno restituant vitalité au texte de l'auteur que Bizet exalte, en l'opposant d'ailleurs au jeu des autres acteurs, incapables de suivre la force vocale de cette actrice, ni d'interpréter et de restituer convenablement au public l'œuvre de Giraudoux.

7 Le concert des voix est donc assez bien présent dans ces comptes rendus, mais que reste-t-il du " paysage sonore ${ }^{15}$ » à l'intérieur duquel l'œuvre était immergée ? Très peu de choses, toute l'attention étant concentrée sur la diction des acteurs, comme si, pour cette civilisation théâtrale, acteur, décor ${ }^{16}$ et texte étaient les seuls éléments de la représentation à analyser et à décrire. Autrement dit, dans ces commentaires, la dimension sonore des spectacles se limite à la rencontre entre les Voix des acteurs et la Voix de l'auteur, cette rencontre étant probablement perçue comme la constituante ontologique du théâtre ${ }^{17}$. Les informations techniques sur le son de cette représentation, par exemple, ne tournent qu'autour du jeu des acteurs. Exactement comme s'il s'agissait d'un spectacle dépourvu de musique de scène, ce qui était le cas, par exemple, des Bonnes ${ }^{18}$. Dans les critiques et dans le programme de ce spectacle, il n'y a en effet aucune trace de musique. En revanche, Jean Maury, dans Combat, décrit de façon détaillée les «mouvements divers de la salle » qui ont suivi la fin de la pièce :

Le spectacle entier se déroule dans un silence à peine murmurant. Mais le rideau tombe. On applaudit, on siffle, on hue. Le rideau se relève. Nouveaux applaudissements mieux marqués, sifflets renforcés (un à roulette quelque part), les « hou! hou !» se transforment en formules plus précises. Troisième lever de rideau. Cette fois, la colère et l'admiration trouvent des mots bien frappés et les deux parties protagonistes de la salle se dressent. Lumière ${ }^{19}$.

Grâce au «scandaleux » Genet, nous avons un compte rendu efficace de la réaction de la salle à cette œuvre. D'abord, nous savons que la pièce a été accueillie dans le silence, qui, au baisser du rideau, se transforme ensuite en voix ${ }^{20}$ préverbales exprimant bien la réception difficile de cette pièce. Enfin, la division de la salle est enregistrée par Maury, les applaudissements et les sifflets relatés avec précision avant que les formules 
d'appréciation ou de dépréciation ne soient exprimées en «mots bien frappés ». Nous avons dans ce commentaire un exemple de réactions sonores, préverbales et ensuite verbales, de l'assistance. Grâce aux sons, le spectateur approuve/désapprouve l'œuvre, en engageant ensuite une "bataille théâtrale ", toute pacifique et sonore, où les voix/ Voix sont des armes - préparées à l'avance, si l'on se fie au sifflet à roulette - pour dominer les adversaires. Ce témoignage, qui indique bien les codes dans lesquels s'exprime la bruyante et rythmique réaction du public, dit surtout que celui-ci, dans une salle de théâtre, constitue une présence complémentaire, une coprésence qui peut devenir active. Nous savons toutefois qu'« au théâtre, la présence est toujours un effet ${ }^{21} "$, soumise par conséquent aux codes d'un medium qui a la caractéristique d'être à la fois ancré dans son temps, "stratégique[ment] » jaloux de son "authenticité », et pourtant à basse « résistance médiatique ${ }^{22}$ ». Le compte rendu journalistique de Maury reconstruit le « paysage sonore » de la fin de la représentation, mais ne s'intéresse pas à celui de la représentation elle-même. Néanmoins, Maury nous permet d'imaginer, presque de visualiser, cette réception si conflictuelle. Les mots de ce critique, plus précisément, s'attèlent à restituer «l'écoute " propre à une salle de théâtre, tout comme sa « réponse » à l'œuvre proposée par l'auteur. Et nous savons que cette écoute «module l'évènement. Pas question de dissocier le visuel et le sonore : au théâtre, le regard du public est audible ${ }^{23}$ ». Par ailleurs, tout comme les enregistrements vidéo de représentations gardent toujours une trace $d u$ «fonctionnement acoustique " du théâtre, pour lequel « le bruit de la salle fait partie du spectacle ${ }^{24}$, de la même manière ce témoignage enregistre la présence acoustique du public.

Quant aux autres critiques, qu'ils aiment ou désapprouvent le texte de Genet, ils trouvent que si le jeu des actrices est presque déclamatoire, leur articulation, elle, est parfaite. Pour ce spectacle aussi, l'interprétation du texte par les actrices en relation au style de l'œuvre dramatique semble constituer le cœur du processus de réception de l'œuvre théâtrale. Remarquons que leur diction est analysée comme détachée de l'« avènement de signification ${ }^{25}$ » qu'est le contenu de la pièce représentée. En pratiquant cette séparation, les critiques semblent pouvoir toujours soit sauver le talent du comédien pour condamner l'auteur, soit le contraire, l'ä $\rho \theta \rho o v$ du langage théâtral - la rencontre entre Voix de l'auteur et des comédiens - étant ainsi analysable en dehors de son contexte d'énonciation ${ }^{26}$.

10 Si avec de telles pièces l'« incarnation » du verbe de l'auteur par le comédien occupe une place centrale dans la presse, il est légitime de s'attendre à ce que celle-ci soit attentive au moindre souffle de ceux qui doivent faire devenir Voix l'œuvre de Molière. Et en effet, pour les représentations de Dom Juan ou Le festin de pierre ${ }^{27}$ et du Tartuffe ou L'Imposteur ${ }^{28}$, nous pouvons presque écouter le « cri de douleur physique ${ }^{29}$ » de Jouvet interprétant Dom Juan, ou encore apprécier la Voix en «faux-bourdon [et le] débit ecclésiastique de paroisse noble ${ }^{30}$ » de son Tartuffe. De la même manière, la sensibilité musicale de Gandrey-Réty le pousse tout naturellement à comparer le jeu de Fernand René (Sganarelle) à l'exécution d'un chanteur lyrique, puisque sa " voix de trial », « qui fausse plutôt le rôle ${ }^{31}$ ", est critiquée avec précision par l'emploi d'un terme technique emprunté au monde de l'opéra ${ }^{32}$. Tandis que grâce à Jacques Lemarchand, nous découvrons que les paysans de Dom Juan parlent avec un accent méridional ${ }^{33}$ - Pol Gaillard précise qu'il s'agit du patois de l'île-de-France avec l'accent de Marseille ${ }^{34}$. Nous pourrions multiplier les traces de l'écoute de la diction des comédiens qui, avec Jouvet, ont donné vie scénique au texte de Molière. Ce qui nous manque dans ces 
descriptions, ce sont à la fois les voix de ces représentations et la musique de Sauguet. En réalité, Jacques Lemarchand, seul parmi les critiques français, la mentionne, mais pour la dénigrer : il affirme en effet qu'elle n'avait même pas «besoin d'être là ${ }^{35}$ », et pose la question suivante :

Est-il juste de conduire vers l'opérette et ses agréables assoupissements la comédie large et profonde qu'est le Tartuffe ? Molière n'a pas passé commande à Lulli quand il s'est mis en tête de monter Tartuffe ${ }^{36}$.

11 Selon le critique, l'œuvre du musicien non seulement est subordonnée à tous les autres éléments de la pièce, elle est même inapte à soutenir la langue de Molière, elle est une intruse dans cette comédie ennoblie par la tradition. Comme si l'aura propre à cette œuvre était offensée par le langage musical de Sauguet, jugé par Lemarchand étranger au registre du Tartuffe, voire vulgaire, et pour cela à rejeter. La réaction de Lemarchand semble non tant attaquer la réalisation de ce musicien en particulier que dénoncer le fait que sa Voix musicale porterait atteinte à l'á $\rho \theta \rho o v$ (l'articulation) propre au théâtre dans le contexte de cette civilisation théâtrale. C'est-à-dire, comme nous l'avons suggéré, l'articulation entre le texte de l'auteur, qui pour ces critiques est sacré, et le jeu des comédiens, dont on attend qu'ils le fassent vivre à travers leurs corps et surtout leurs Voix.

12 À la lecture certes "sélective » de ces critiques, nous pourrions imaginer que la voix, dans ces productions théâtrales, était négligée. Bruno Sebald et Marie-Gabrielle Soret ont au contraire souligné l'attention apportée par Jouvet à la dimension sonore du théâtre. Ayant étudié « [l]es archives sonores du fonds Jouvet », ils affirment que

Jouvet, acteur, metteur en scène, professeur, théoricien, était extrêmement sensible au monde sonore tout autant qu'au visuel et, en considérant les archives de ce point de vue, on y trouve quantité d'éléments sonores, bien souvent transposés en éléments musicaux [...]. Tous éléments qui font partie du travail quotidien de l'acteur et de l'apprentissage des bases de son métier, et appartiennent autant au champ de la déclamation dramatique qu'à celui de la déclamation lyrique ${ }^{37}$.

Pour ce pédagogue et metteur en scène, en effet, «le théâtre c'est d'abord un exercice de diction, qui est équivalent au pétrissage. Quand [...] cette substance dramatique est bien assimilée [...], quand on l'a réduite par la bouche et les poumons, je vous assure qu'on arrive à un sentiment ${ }^{38}$ ». Les critiques semblent connaître le travail technique par lequel le texte est manié afin que le contenu émotionnel de l'œuvre représentée ou l'«esprit» du personnage interprété surgissent ${ }^{39}$. Ce qui est remarquable dans ce passage, c'est qu'il permet de mettre en évidence le fait que Jouvet conseille à ses élèves d'arriver à un sentiment via une technique vocale : c'est d'abord par un » exercice de diction» qu'on a la certitude de trouver le sentiment, de toucher l'esprit de la «substance dramatique». Autrement dit, la Voix de chaque acteur, en tant qu'instrument et avant que celui-ci ne soit porteur d'un contenu, est le principal véhicule entre l'œuvre et sa représentation, entre le personnage et le comédien. De ce point de vue, Jouvet aussi serait le témoin d'une civilisation où la Voix est l'architrave du système théâtral dans sa globalité, pierre de touche de la bonne ou de la mauvaise réussite d'un spectacle.

14 Marie-Gabrielle Soret souligne cependant que pour la conception des musiques de Dom Juan, Jouvet avait commencé, dès 1946, à collaborer intensément avec Igor Markevitch ${ }^{40}$, et que cette collaboration avait pour but de réaliser quelque chose de similaire à un "décor sonore" ante litteram. Grâce à l'étude menée par cette musicologue, nous prenons connaissance du fait que les deux artistes avaient élaboré 
les notions de «musique ", "semi-musique » et «bruit de fond " afin d'indiquer dans le détail le type d'effet sonore à produire à chaque musique de scène. Les différents types de «tonnerre », par exemple - qui varient du plus « imitatif » au « plus musical» ou au "plus musical encore $»^{41}-$, nous indiquent avec précision que l'enregistré doit se fondre dans les sons live, en contribuant de façon structurelle à trouver et à soutenir le rythme de l'œuvre. Cette collaboration ne pourra finalement avoir lieu et Jouvet choisit alors de s'appuyer sur le fidèle Sauguet. S'il nous paraît légitime d'imaginer que certains éléments de cette conception musicale aient pu être repris, nous ignorons toutefois si Jouvet a finalement renoncé à donner un caractère réellement novateur à la musique de scène de Dom Juan. En tout cas, les critiques ne parlent que très peu de la réalisation sonore de ce spectacle. Sebald cite toutefois un passage d'un hommage de Sauguet à Jouvet, dans lequel le musicien affirme que le choix de Jouvet de se servir principalement de musiques enregistrées s'expliquerait aussi bien par des raisons matérielles que par des raisons d'ordre esthétique. Sauguet y explique précisément qu'« une fois enregistrée et mise en disques, la musique lui appartenait [il parle de Jouvet], lui apparaissait plus soumise à son dessein, à son travail, à son but $[. . .]^{42}$ ", ce qui indiquerait que la musique occupait une place stratégique dans la pratique opératoire concrète du metteur en scène, quoique «soumise à son dessein ». En effet, Sauguet souligne en premier lieu que si, pour Jouvet, le décor est central et le rapport entre lui et Bérard unique, privilégié, la musique, elle, est «affaire » et propriété de Jouvet, le musicien réalisant ce qui appartient au metteur en scène : donner le rythme, faire émerger la musique du texte. En second lieu, les mots de Sauguet témoigneraient du rôle primordial de la musique à l'intérieur du processus de création des spectacles de Jouvet, puisque celle-ci structure la totalité de la représentation, jusqu'à accompagner et soutenir la réception de l'œuvre par le spectateur ${ }^{43}$. Bruno Sebald a par ailleurs sans aucun doute raison de souligner que «l'étude [de l'archive sonore du fonds Louis Jouvet] permet de dégager un certain nombre de constats sur l'importance qu'accordait Jouvet à l'enregistrement sonore ${ }^{44}$ ». Ses analyses, de plus, montrent "l'importance de[s musiques de scène enregistrées et ensuite mises en disque] dans le déroulement ${ }^{45} »$ des pièces représentées par Jouvet. Sauguet affirme clairement, néanmoins, que si le but des mises en scène de Jouvet était bien celui « de faire vivre, d'exalter [...] l'œuvre et la pensée du dramaturge », la musique, certes, participait à "cette sorte de cérémonie", "à un degré moindre peut-être [que le décor, les éclairages, les costumes], mais non moins essentiellement ${ }^{46} »$.

15 Le témoignage de Sauguet nous paraît précieux dans la mesure où il restitue à notre contemporanéité le point de vue d'un musicien, d'un collaborateur de Jouvet, d'un homme donc activement impliqué dans le contexte de sa propre civilisation théâtrale, pour qui la musique de scène serait hiérarchiquement soumise aux autres composants de la représentation ${ }^{47}$. Sauguet précise en effet que le rôle du "musicien [dans la pratique de Jouvet] était moindre et plus accessoire ${ }^{48} »$. Dans le même temps, Sauguet ne cache pas que la musique « apportait un des éléments essentiels ${ }^{49}$ » aux spectacles de Jouvet. À la fois "soumise à son dessein ", et centrale dans sa pratique de metteur en scène, le rôle de la musique - et du son aussi, au vu du travail entamé avec Markevitch - serait pour Jouvet stratégique dans la mesure où celle-ci donnerait une structure au travail des Voix des acteurs confrontées à celle de l'auteur. Si bien que, la musique étant nécessairement soumise à la synthèse harmonique de tous les éléments de la représentation, elle pourrait être perçue par Jouvet comme sa propriété, dès lors que celui-ci a en premier lieu le devoir de trouver et de concerter toutes les voix/Voix de la 
représentation. Pour cette même raison, le son semble jouer un rôle « essentiel » dans sa praxis.

La place quelque peu ancillaire que la réalisation sonore aurait eue chez Jouvet, selon ce qu'en a dit Sauguet, aurait pu être remise en question - ou confirmée - si la collaboration avec le compositeur russe avait eu lieu ${ }^{50}$. L'hypothèse selon laquelle la civilisation théâtrale ici évoquée - celle où Jouvet produit ses œuvres et la critique ses analyses - serait dominée, du moins d'un point de vue acoustique, par la rencontre des Voix ne nous semble pas moins saisissante. Seul un travail rigoureux sur toute cette période, étendu à plusieurs théâtres et fondé sur un corpus de critiques plus ample, pourrait valider ou infirmer les hypothèses que nous avons essayé d'ouvrir et de formuler. Nous nous limiterons pour notre part dans le cadre de cette étude à une présentation des traces du sonore dans les autres critiques consultées, ce qui nous permettra de prolonger les pistes de recherche proposées.

La première pièce que nous rencontrons sur notre chemin d'enquête pour la période qui a suivi la mort de Jouvet, alors que la direction de l'Athénée était assurée par Françoise Grammont, est Cher menteur ${ }^{51}$. Les interprètes qui donnent Voix à cette œuvre sont Maria Casarès et Pierre Brasseur. Bertrand Poirot-Delpech affirme que devant leur jeu

on pourrait presque fermer les yeux. On devrait même le faire [...]. Ce qui compte c'est le plaisir de l'oreille. Il faudrait le vocabulaire d'un critique musical pour parler d'une rencontre de voix comme celles de Brasseur et de Maria Casarès. L'une tient ses notes de basse chantante à pleine gorge, tandis que l'autre, semblant par contraste moins ténébreuse, fait vibrer ses timbres de hautbois. [...] La musique porte la marque de Jean Cocteau - auteur de la version française - qui connaît bien les ressources de sa chère Casarès ${ }^{52}$.

18 Le critique du Monde ne cache pas son admiration pour le jeu et la maittrise vocale de ces deux comédiens, suivi en cela par tous ses collègues dans les commentaires consultés. Pour Paul Morelle, ce spectacle «[...] n'est pas du théâtre. C'est un double récital, un duo, un poème récité à deux voix ${ }^{53}$ ». Et le compositeur est Cocteau, auteur/ traducteur qui a écrit sa musique scénique en fonction de la Voix d'une comédienne aimée. Avec cette œuvre, l'hypothèse d'une civilisation théâtrale dominée par la Voix semblerait être confirmée, quoiqu'une analyse du spectacle devrait permettre de vérifier sa particularité dans le contexte du théâtre des années 1950 et 1960. Essayons de poser une simple question. En 1963, Françoise Spira, qui un an plus tôt a pris la direction de l'Athénée, intègre à l'équipe technique de son Théâtre vivant un responsable de la fonction "Recherche et sonorisation », Michel Marcland. Nous savons qu'en 1965-1966, l'équipe comporte un "Service des recherches", confié à Alain Resnais ${ }^{54}$, et un "Conseiller musical », Diego Masson ${ }^{55}$. Le travail de ces techniciens estil représenté dans les analyses des critiques dramatiques? Ou bien le ton et l'argument de ces commentaires seront-ils comparables à ceux de Poirot-Delpech pour le travail du duo Casarès/Brasseur?

Pour le texte de Marguerite Duras La Bête dans la jungle ${ }^{56}$, il n'y a pas de doute : l'écoute de cette représentation est dominée par la musique vocale des interprètes en scène $\mathrm{e}^{57}$, les voix de ce spectacle sont absentes. Alors que pour La Brigitta de Jacques Audiberti ${ }^{58}$, spectacle interprété par la compagnie Le théâtre vivant, quelque chose semble changer. En effet, Paul Morelle, attaquant « le théâtre parisien [qui serait] arrivé à un tel degré de paroxysme exacerbé et incompréhensible [...] que la seule attitude raisonnable et charitable à son égard semblerait devoir être le silence", ne tait pas pourtant la 
dimension sonore générale de cette production, bien qu'il juge qu'il soit « impossible de s'intéresser à ces cris, à ces pétarades, à toute cette agitation incohérente et démentielle, à ces délires de mots torrentiels et décousus qui se répandent comme une inondation $~_{59}$. Or, bien que ces voix soient décrites pour être proscrites, derrière «ces pétarades » il est possible d'écouter : « [l]a motocyclette [qui] fait entendre des bruits étranges comme ceux qui accompagnaient autrefois une pièce d'avant-garde de Boris Vian $»^{60}$. Ici, enfin, les voix d'un spectacle parlent, tout comme la réaction de Morelle signifie la résistance de ce spectateur à des nouveaux phénomènes acoustiques et au caractère "avant-gardiste » que l'auteur du texte et les interprètes semblent avoir donné à cette œuvre. Aucune trace, toutefois, du travail de Fred Kiriloff qui, assisté de Michel Marcland, a réalisé le son de ce spectacle. Kiriloff est pourtant le pionnier de la création sonore théâtrale en France, celui qui depuis 1952 collabore avec d'importants théâtres et productions, réalisant divers "décors sonores " ${ }^{61}$. En 1973, lors de la représentation du Borgne ${ }^{62}$, il sera salué par la presse comme le «meilleur spécialiste aujourd'hui (que tout le monde s'arrache, du théâtre d'avant-garde à la ComédieFrançaise) $)^{63}$ ». Pour ce spectacle, il réalisera une « sonnerie prodigieuse de trompettes, les rugissements terribles des lions que l'on sent tout proches ", qui, «mêlés à l'on ne sait quel hurlement démentiel ${ }^{64} »$, font toute la particularité de cette œuvre. De laquelle, par ailleurs, disparaissent les Voix des acteurs en tant que telles, la réception du son se concentrant sur l'« excellente réalisation sonore - ici, très importante - de Fred Kiriloff ${ }^{65}$ ». Si, au milieu des années 1970, Kiriloff est déjà connu, en 1962, son travail semble sous-représenté dans la presse - à l'instar de celui de ses collègues Diego Masson, qui adapte en 1963 les « airs folkloriques » de La Danse du sergent Musgrave ${ }^{66}$, et Pierre Boeswillwald ${ }^{67}$, réalisateur de la tempête qui, dans la pièce Après la pluie $e^{68}$, en 1968, frappe le critique Claude Megret $^{69}$.

Ajoutons seulement, pour conclure, un simple constat. Si le travail de ces techniciens n'apparaît pas dans les critiques des années 1960 consultées, les Voix de Laurent Terzieff et de Laurence Bourdil, interprètes de La Danse du sergent Musgrave, ne sont pas non plus évoquées ${ }^{70}$. Si nous observons que pour des productions telles que Les Séquestrés d'Altona en 1965 ou encore Irma la Douce en 1967, les Voix des comédiens sont prépondérantes et semblent encore décider de la réussite d'un spectacle, nous voudrions poser une dernière question et essayer d'ouvrir une dernière piste de recherche. Le caractère quelque peu expérimental de la mise en scène de Peter Brook ne convainc pas unanimement la critique, qui décrète plutôt la non-réussite de la pièce. Pour quelle raison les Voix de ce spectacle disparaissent-elles pour faire place aux voix des tambours et de la danse ? Sommes-nous face à un changement de paradigme dans l'écoute du théâtre ou tout simplement la critique a-t-elle été assourdie par les bruits et par le jeu des acteurs dirigés par Brook? Si une question de ce genre ne paraît pas oiseuse, alors une étude qui mettrait en évidence les changements de l'attention du public - de la critique en particulier - envers le son, tout comme l'atténuation de la résistance médiatique envers les innovations, se révélerait fructueuse. Une telle recherche pourrait, par exemple, élargir son champ d'enquête en augmentant ses sources, pour les croiser ensuite avec l'histoire de compagnies et de théâtres sur lesquels elle choisirait de se concentrer. En étudiant aussi la pratique d'hommes de théâtre tels que Kiriloff, il serait en outre possible de mieux comprendre le contexte dans lequel ils ont opéré, en essayant de mettre en lumière l'évolution des recherches dans le champ de l'acoustique. De ce point de vue, une étude du Traité de prise de son ${ }^{71}$ de José Bernhart pourrait se révéler productive, afin de reconstituer la pratique 
opératoire de Kiriloff. Ce dernier parle en effet de cet ouvrage comme du manuel de référence pour son métier ${ }^{72}$. Il serait intéressant de chercher à savoir, ensuite, si les critiques ont enregistré les traces du travail de Kiriloff. Enfin, il serait souhaitable de comparer, à l'intérieur des comptes rendus consultés, la présence des traces du sonore avec celles du visuel, en menant une approche à la fois qualitative et quantitative.

\section{NOTES}

1. Les dossiers de presse de la Bibliothèque nationale de France (recueils factices de coupures de presse) sont consultables au département des Arts du spectacle de la BnF (site Richelieu).

2. À savoir, les années qui ont suivi la Seconde Guerre mondiale jusqu'à la mort de Louis Jouvet en 1952, les années 1960, et le passage des années 1960 aux années 1970.

3. «Les sources sont sources quand quelqu'un les regarde historiquement. » Douze leçons sur l'histoire, Paris, Seuil, [1996] 2010, p. 80.

4. "À l'époque de la reproduction technologique, nous pouvons parfois faire l'expérience d'un passé audible, mais nous ne pouvons que présumer l'existence d'un passé auditif. » Jonathan Sterne, "Le passé audible », traduction de Françoise Ouellet, Le Son du théâtre. 1. Le passé audible, Théâtre/Public, n 197, juillet-septembre 2010, p. 18.

5. Jean-Marc Larrue et Marie-Madeleine Mervant-Roux, "Théâtre: le lieu où l'on entend", L'Annuaire théâtral, n 56-57, Montréal, automne 2014-printemps 2015, p. 22.

6. Dans l'article "Corps et âmes, présences et absences", Le Son du théâtre. 1. Le passé audible, Théâtre/Public, n 197, op. cit., p. 98-106. Le philosophe italien Giorgio Agamben approfondit la question du langage et de son statut ontologique dans l'ouvrage Le Langage et la mort. Un séminaire sur le lieu de la négativité, trad. de Marilène Raiola, Paris, Christian Bourgois, " Détroits », 1991 [Il linguaggio e la morte, Turin, 1986]. L'auteur y affirme que «[1]'avoir-lieu du langage entre la suppression de la voix et l'avènement de signification est l'autre Voix, [...] qui, dans la tradition métaphysique, constitue l'articulation originelle (l'ó $\rho \theta \rho o v)$ du langage humain. Mais, en tant que cette Voix (que nous écrirons désormais avec une majuscule pour la distinguer de la voix comme simple son) a le statut d'un non-plus (voix) et d'un non-encore (signifié), elle constitue nécessairement une dimension négative. Elle est le fondement, mais au sens où elle est ce qui s'abîme et disparaît, afin que l'être et le langage aient lieu ", ibid., p. 74-75 (souligné par l'auteur). Sans que notre réflexion s'inscrive dans la profondeur ontologique du discours d'Agamben, l'usage de ces catégories aura du moins l'effet, nous l'espérons, de suggérer l'importance de toutes les voix/Voix émises par et dans le medium théâtre, tout en suggérant la spécificité irréductible de chacune d'entre elles.

7. Pour approfondir cette notion, voir l'ouvrage des historiens du théâtre Fabrizio Cruciani et Clelia Falletti (dir.), Civiltà teatrale nel xxe secolo, Bologne, Il Mulino éditeur, 1986.

8. Mise en scène : Louis Jouvet; décors et costumes : Christian Bérard; musique : Henri Sauguet. La répétition générale, ouverte à la critique, a fort probablement eu lieu le 19 décembre 1945.

9. Recueil d'articles : LJ SW 12 (1-2-3).

10. Ibid.

11. Ibid., Paris-Presse, 2 janvier 1946.

12. Ibid. 
13. Gaston Boyer, Libération Soir, 21 décembre 1945, ibid. Il conviendra de remarquer que ce critique pour Giraudoux parle de "génie", caractéristique qui manque à Jouvet ou au scénographe Christian Bérard : ces derniers «n'ont que du talent », alors que Giraudoux sera joué lorsque ces hommes de théâtre n'existeront plus. Et il ajoute : «ils sont remplaçables, lui il est unique ». Mais le fait d'avoir servi fidèlement l'auteur « sera leur gloire ».

14. Jean Gandray-Réty, Arts, 21 décembre 1945, ibid. D'ailleurs, selon Jean-Pierre Dorian, Les Arts et Lettres, 2 janvier 1946, le «Mozart qui a[vait] pu tresser ces guirlandes de considérations sur l'amour, la mort, les affaires, le pétrole, les syndicats, le cœur, [...] le musicien qui en avait conçu la musique ", c'est l'auteur du texte.

15. Sur l'importance de ce concept à l'intérieur des Sound Studies, voir Jonathan Sterne, «Les espaces stéréophoniques du paysage sonore ", in Le Son du théâtre (XIX ${ }^{e}$ XXI ${ }^{e}$ siècle), Jean-Marc Larrue et Marie-Madeleine Mervant-Roux (dir.), Paris, CNRS Éditions, 2016, p. 37-56. La riche étude de Sterne reprend, approfondit et développe le concept de soundscape à partir de la définition que R. Murray Schafer propose de ce terme dans l'ouvrage Le Paysage sonore. Toute l'histoire de notre environnement à travers les âges, trad. Sylvette Gleize, Paris, J.-C. Lattès, [1977] 1979. À partir de l'étude de Carlotta Darò, Avant-gardes sonores en architecture, Dijon, Les presses du réel, 2012, Sterne affirme que dans l'article de Richard Buckminister Fuller, «The Music of the new Life» (Music Educators Journal, vol. 52, $\mathrm{n}^{\circ} 6-7,1966$ ), nous pouvons trouver la première utilisation de ce terme dans toute sa complexité. Mentionnons aussi, dans le contexte français, l'étude d'Alain Corbin, Les Cloches de la terre. Paysage sonore et culture sensible dans les campagnes au XIX ${ }^{e}$ siècle, Paris, Albin Michel, 1994, ainsi que celle de Jean-Pierre Gutton, Bruits et sons dans notre histoire, Paris, Presses universitaires de France, 2000. Ce second ouvrage s'appuie lui aussi sur la définition de "paysage sonore » telle qu'elle a été développée par Murray Schafer. Enfin, l'article de Marie-Madeleine Mervant-Roux, « De la bande-son à la sonosphère. Réflexion sur la résistance de la critique théâtrale à l'usage du terme "paysage sonore" ", Images Re-vues [en ligne, consulté le $1^{\text {er }}$ octobre 2018, URL : http://imagesrevues.revues.org/428], permet de retracer l'histoire de la méfiance, puis de l'usage particulier qui a conduit la critique professionnelle française à prendre en considération ce terme, tout en l'adaptant au contexte théâtral.

16. S'il n'y a en effet que très peu de place dans la presse pour le travail de Sauguet, celui de Bérard a une importance quasi égale au travail de Jouvet, les deux restant «soumis" au magistère de l'auteur.

17. Voir Jean-Marc Larrue, «Son, présence et ontologie. Perspectives intermédiales sur le son du théâtre ", in Le son du théâtre (XIX ${ }^{e}-\mathrm{XXI} I^{e}$ siècle), op. cit., p. 215-234.

18. Texte de Jean Genet; mise en scène de Louis Jouvet; décors de Christian Bérard; décors meublés de Jacques Damiot (sous la direction de Christian Bérard) ; costumes de Jeanne Lanvin. Première le 19 avril 1947. Recueils d'articles : LJ-501-26 (1-2) et LJ-MS-142 pour la distribution.

19. « Nervosité au Théâtre de l'Athénée », Combat, 28 avril 1947.

20. En reprenant la distinction agambienne.

21. Jean-Marc Larrue, «Son, présence et ontologie. Perspectives intermédiales sur le son du théâtre », op. cit., p. 229.

22. Comme les études de Jean-Marc Larrue l'ont démontré.

23. Marie-Madeleine Mervant-Roux, «De la bande-son à la sonosphère. Réflexion sur la résistance de la critique théâtrale à l'usage du terme "paysage sonore" », op. cit. Mervant-Roux souligne que «[1]'aire de jeu est inscrite dans la cité, dont les spectateurs sont les représentants, et le spectacle ne peut fonctionner que sur la base d'un accord minimal entre la ville et le groupe des acteurs. Cette mutualité - qui n'a rien d'une communion, puisqu'elle est parcourue de conflits, de tensions, de passions et de rires - peut être saisie visuellement (surtout avant le début du spectacle), mais c'est acoustiquement qu'elle s'exprime et qu'elle est agissante. L'écoute, surtout muette, est la réponse de la salle. Si les conventions ordonnant les comportements des spectateurs évoluent au cours de l'histoire (imposition du silence, règles régissant les bravos, 
techniques pour limiter les bruits intempestifs...) et varient selon les styles de spectacles, la rumeur du théâtre ("Theatre Noise"), avant, pendant, après la performance, possède des traits remarquables. »

24. Marie-Madeleine Mervant-Roux, L'Assise du théâtre. Pour une étude du spectateur, Paris, CNRS Éditions, 1998, p. 170.

25. Giorgio Agamben, Le Langage et la mort. Un séminaire sur le lieu de la négativité, op. cit., p. 74.

26. Parler de Voix à propos de l'auteur de l'œuvre dramatique et à propos du comédien qui l'interprète revient à souligner la valeur technique de cette rencontre, antérieure dans le processus de préparation de l'acteur pour Louis Jouvet, séparée du contenu de la production théâtrale dans le jugement de la critique.

27. Première le 24 décembre 1947. Musique d'Henri Sauguet; décors et costumes de Christian Bérard.

28. Première le 27 janvier 1950. Musique d'Henri Sauguet; décors et costumes de Georges Braque.

29. Grâce à un compte rendu anonyme, publié dans Carrefour, le 31 décembre 1947. Recueil d'articles : 8-RSUPP-2264.

30. Robert Kemp, Le Monde, 27 janvier 1950. Recueil d'articles : LJ-SW-16.

31. Jean Gandrey-Réty, Franc-Tireur, 25 décembre 1947. Recueil d'articles : 8-RSUPP-2264.

32. Le mot trial désigne le timbre de voix d'un ténor « s'employant aussi à traduire le comique ou le populaire ». Sophie Comet, Denis Morrier, « Vocale (Distribution) », Encyclopcedia Universalis [en ligne, consulté le 28 septembre 2018].

33. Combat, 26 décembre 1947.

34. Les Lettres Françaises, $1^{\mathrm{er}}$ janvier 1948.

35. Combat, 22 janvier 1950. Recueil d'articles : LJ-SW-16.

36. Ibid.

37. «Les archives sonores du fonds Jouvet ", Le Son du théâtre. 2. Dire l'acoustique, Théâtre/Public, $\mathrm{n}^{\circ} 199,1^{\mathrm{er}}$ trim. 2011, p. 26.

38. Louis Jouvet, "Alceste. Classe du 30 avril 1940 ", in Molière et la comédie classique, Paris, Gallimard, 1965, p. 15. Les passages sont soulignés par l'auteur.

39. Notons, par exemple, le précieux témoignage de Jean Duché, qui, dans Le Figaro littéraire du 28 janvier 1950, affirme que les comédiens ont donné les vers du Tartuffe « tout simplement dans la respiration ». Grâce aux confidences de l'interprète de Valère (Jean Richard), Duché révèle aussi que Jouvet a obligé les acteurs à « parler longtemps le texte avant de les autoriser à trouver la rime ", du moins pour ce qui concerne cette mise en scène-là.

40. D'origine russe, Igor Markevitch (1912-1982) fut l'un des plus jeunes et prometteurs compositeurs et chefs d'orchestre de sa génération. Il commença sa carrière avec les Ballets russes de Diaghilev à l'âge de 16 ans.

41. Bruno Sebald et Marie-Gabrielle Soret, op. cit., p. 25-26.

42. Henri Sauguet, «Louis Jouvet et la musique ", Louis Jouvet 1887-1951. Notes et documents, Revue de la Société d'histoire du théâtre, $4^{\mathrm{e}}$ année, $1^{\mathrm{er}} 2^{\mathrm{e}}$ trim., 1952, p. 146-147.

43. «Pour rendre plus intense l'atmosphère ou le décor, pour aider le comédien dans son jeu, dans sa démarche, dans sa plus complète expression, pour narguer ou pour émouvoir, pour ne pas laisser le spectateur se reprendre entre les différents tableaux d'une même œuvre, pour le préparer à entendre ou pour souligner quelque effet scénique, Louis Jouvet attendait de la musique qu'elle l'aidât, - et aussi pour aller jusqu'à l'accomplissement parfait de l'harmonie du spectacle qu'il avait élaboré. » Ibid, p. 145.

44. Bruno Sebald et Marie-Gabrielle Soret, op. cit., 18.

45. Ibid, p. 24.

46. Henri Sauguet, « Louis Jouvet et la musique », op. cit.

47. En ce qui concerne la pratique opératoire de Louis Jouvet, du moins. Cela étant dit, il est nécessaire de souligner que les affirmations de ce compositeur devraient être vérifiées par une 
étude plus approfondie de son rapport avec Jouvet, de son implication dans le contexte théâtral de son époque et dans celui de la compagnie du Théâtre de l'Athénée.

48. Henri Sauguet, op. cit., p. 147.

49. Ibid.

50. Seul un travail mené simultanément à l'intérieur et autour du fonds Louis Jouvet pourrait permettre de vérifier la réelle importance de la musique, ainsi que son statut, dans l'ensemble de la pratique théâtrale du directeur de l'Athénée.

51. Auteur et metteur en scène : Jérome Kilty ; traduction de Jean Cocteau. Première le 5 octobre 1960.

52. Le Monde, 6 octobre 1960. Recueil d'articles : 8-SW-217.

53. Libération, 6 octobre 1960.

54. Il faut rappeler que Françoise Spira avait joué dans le film L'Année dernière à Marienbad.

55. Fondateur, en 1966, de l'ensemble Musique vivante, avec Luciano Berio et Pierre Boulez, Diego Masson est un directeur d'orchestre de premier plan dans le panorama musical européen.

56. Mise en scène de Jean Lauvrais et de François Maistre; dispositif scénique et costumes d'André Acquart. Première le 12 septembre 1962.

57. Voir le recueil d'articles : 8-SW-605.

58. Mise en scène de François Maistre et Jean Larroquette ; costumes d'André Acquart. Première le 28 septembre 1962 .

59. Libération, « Pour une critique du silence ", 9 septembre 1962. Recueil d'articles : 8-SW-607.

60. Jean Paget, Combat, date non renseignée, ibid.

61. Voir : http://www.regietheatrale.com/index/index/donateurs/Fred-Kiriloff.html. [Consulté le 15 mai 2018].

62. Pièce d'Eduardo Manet; mise en scène de Michel Fagadau; décors et costumes de Hubert Monloup.

63. Jean Mambrino, Études, décembre 1973. Recueil d'articles : 4-SW-9804.

64. Ibid.

65. Jean-Jacques Gautier, Le Figaro, 19 octobre 1973.

66. Mise en scène de Peter Brook; décors et costumes de Peter Brook et Adèle Hankey. Spectacle produit par Le théâtre vivant. Première le 2 octobre 1963.

67. Pionnier de la musique électroacoustique, ce compositeur se consacre, dès 1954, à des expérimentions sonores dans un contexte théâtral, pour se concentrer, à partir de 1968, à l'étude de la musique fondamentale avec Pierre Schaeffer.

68. Mise en scène de René Dupuy; décors et costumes de Jacques Le Marquet. Première le 3 février 1968.

69. Carrefour, 14 février 1968. Recueil d'articles : 4-SW-2378.

70. Voir recueil d'articles : 4 -SW-1.

71. Paris, Eyrolles, 1949.

72. Dans l'article de Marcel Marcland, «Le décor sonore: art ou technique", Théâtre. Drame, musique, danse, $\mathrm{n}^{\circ} 27$, Paris, janv. 1962, p. 8-9. 


\section{RÉSUMÉS}

La présente étude fait suite à un travail accompli en juin 2017 au sein du projet ECHO, consacré à l'évaluation de la place du son et de l'acoustique dans les critiques dramatiques d'une sélection de spectacles mis en scène au Théâtre de l'Athénée, de 1945 à 1973. Nous référant à l'historien Antoine Prost, selon lequel «les sources sont sources quand quelqu'un les regarde historiquement ", il s'agit ici de regarder "historiquement» les traces du son et de l'auralité présentes dans ces textes. Si par «auralité ", on entend la "combinaison de l'audible et de l'auditif » et son inscription dans des contextes culturels et historiques définis, nous essayerons d'écouter ces documents textuels en tant que témoins de la dimension sonore des œuvres dont ils parlent. Nous formulerons ensuite des hypothèses concernant la qualité et l'évolution de cette écoute.

The present research continues a work completed in June 2017 as part of the ECHO project, which was devoted to identifying the importance of sound and acoustics in the theatre criticism of a selected number of plays staged at the Théatre de l'Athénée between 1945 and 1973. Consistent with the historian Antoine Prost, who states that "sources are sources if someone looks at them historically", this article takes a "historical" look at the traces of sound and aurality to be found in these texts. If the notion of aurality is taken to signify "the combination of the audible and the auditory" and its place within defined cultural and historical contexts, we listen to these textual documents as testaments to the sound dimension of the works they address. Different hypotheses regarding the quality and evolution of this listening are then proposed.

\section{INDEX}

Keywords : Sound Studies, Théâtre de l'Athénée, theatre criticism, music, sound design, stage direction

Mots-clés : Sound Studies, Théâtre de l'Athénée, critique dramatique, musique, décor sonore, mise en scène

\section{AUTEUR}

\section{TOMMASO ZACCHEO}

Tommaso Zaccheo a soutenu en juin 2017 un mémoire de Master 2 intitulé « Roger Planchon et la notion d"“écriture scénique" (1957-1962) ", dirigé par Marco Consolini. Actuellement doctorant auprès de l'École doctorale 267-Arts et médias, il travaille à une thèse intitulée « Roger Planchon et ses théâtres (1957-1987) », toujours sous la direction de Marco Consolini. Il est en outre chercheur associé auprès du département des Arts du spectacle de la Bibliothèque nationale de France, où il mène un travail à la fois de recherche et archivistique intitulé « L'œuvre de Planchon et de ses collaborateurs au Théâtre national populaire à Villeurbanne ». Sa collaboration avec le projet ECHO a débuté en juin 2017 et a principalement été consacrée à l'écoute des témoignages concernant le son et à l'auralité telle qu'elle peut être saisie dans les critiques dramatiques. 